\title{
Arterial Spin-Labeling Perfusion for PHACE Syndrome
}

\author{
(D) M.D. Mamlouk, (D) A. Vossough, (D) L. Caschera, DM. Maheshwari, and (D)C.P. Hess
}

\begin{abstract}
BACKGROUND AND PURPOSE: Arterial stroke is a rare-but-reported complication in patients with posterior fossa brain malformations, hemangiomas, arterial anomalies, coarctation of the aorta and cardiac defects, and eye abnormalities (PHACE) syndrome. Currently, stroke risk is inferred by the severity of arterial anomalies identified on MRA, though no evidenced-based data exist. The purpose of our study was to determine whether arterial spin-labeling MR imaging perfusion can detect alterations in CBF in patients with PHACE syndrome.
\end{abstract}

MATERIALS AND METHODS: Records were reviewed from 3 institutions for all patients with PHACE syndrome who underwent arterial spin-labeling from 2000 to 2019. CBF was qualitatively investigated with arterial spin-labeling to determine whether there was decreased or normal perfusion. Arterial anomalies were characterized on MRA imaging, and parenchymal brain findings were evaluated on conventional MR imaging sequences.

RESULTS: Forty-one patients with PHACE syndrome had arterial spin-labeling imaging. There were 30 females and 11 males (age range, 7 days to 15 years). Of the 41 patients, 10 (24\%) had decreased CBF signal corresponding to a major arterial territory. Ten of 10 patients had decreased CBF signal in the anterior circulation, 2/10 had decreased anterior and posterior circulation CBF signal, 2/10 had decreased bilateral anterior circulation CBF signal, and 1/10 had globally decreased CBF signal. Forty of 41 (97.5\%) patients had at least 1 arteriopathy, and in those with decreased CBF signal, the arteriopathy corresponded to the CBF signal alteration in 10/10 patients.

CONCLUSIONS: Arterial spin-labeling can potentially characterize hemodynamic changes in patients with PHACE syndrome.

ABBREVIATIONS: ASL = arterial spin-labeling; PHACE = posterior fossa brain malformations, hemangiomas, arterial anomalies, coarctation of the aorta and cardiac defects, and eye abnormalities

A rterial ischemic stroke is a rare-but-devastating complication in a minority of patients with posterior fossa brain malformations, hemangiomas, arterial anomalies, coarctation of the aorta and cardiac defects, and eye abnormalities (PHACE) syndrome. Stroke has been reported in multiple patients with PHACE syndrome, ${ }^{1-6}$ but the etiology of stroke is poorly understood. ${ }^{1}$ Possible mechanisms for stroke with PHACE include the following: 1) artery-to-artery embolisms, 2) ischemia from reduced blood flow, or 3) cardioembolism. These etiologies are

Received May 28, 2020; accepted after revision August 19

From the Department of Radiology (M.D.M.), The Permanente Medical Group, Kaiser Permanente Medical Center, Santa Clara, Santa Clara, California; Department of Radiology and Biomedical Imaging (M.D.M., C.P.H.), University of California, San Francisco, San Francisco, California; Department of Radiology (A.V., L.C.), Children's Hospital of Philadelphia, Perelman School of Medicine, University of Pennsylvania, Philadelphia, Pennsylvania; Department of Radiology (L.C.), La Fondazione Institute for Research, Hospitalization and Health Care Ca' Granda Ospedale Maggiore di Milano Policlinico, Milan, Italy; and Department of Radiology (M.M.), Medical College of Wisconsin, Children's Hospital of Wisconsin, Milwaukee, Wisconsin.

Paper previously presented as an abstract at: International Society and Study of Vascular Anomalies Workshop, May 14-15, 2020; Virtual. predicated on arteriopathies in the brain, neck, and aortic arch, which are the most common extracutaneous finding in patients with PHACE syndrome. ${ }^{7}$ In 1 study, arteriopathies were observed in $91 \%$ of 33 patients $^{8}$ and ranged from an anomalous course to marked stenosis with a Moyamoya pattern. ${ }^{3}$ Currently, stroke risk is only inferred by the severity of these arteriopathies identified on MRA imaging. ${ }^{9}$ Despite knowledge of the types of arteriopathies in $\mathrm{PHACE},{ }^{3}$ it is unclear why certain patients with arteriopathies experience a stroke and others do not, even if there are severe anomalies in both subsets. To date, no evidence-based data exist on stroke risk in PHACE syndrome.

\footnotetext{
Please address correspondence to Mark D. Mamlouk, MD, Department of Radiology, The Permanente Medical Group, Kaiser Permanente Medical Center, Santa Clara, 700 Lawrence Expy, Santa Clara, CA 95051; e-mail: mark.d.mamlouk@kp.org, mark.mamlouk@ucsf.edu; @MarkMamloukMD

--.- Indicates open access to non-subscribers at www.ajnr.org

$\equiv$ Indicates article with supplemental online table.

http://dx.doi.org/10.3174/ajnr.A6871
} 
Arterial spin-labeling (ASL) is a noncontrast MR imaging perfusion sequence that has been studied in patients with stroke and vasculopathies, particularly with the Moyamoya pattern and has proved useful in analyzing $\mathrm{CBF} .{ }^{10,11}$ In the setting of PHACE syndrome, the concept of ASL imaging has been introduced ${ }^{12,13}$ as well as for other cutaneous vascular anomalies. ${ }^{12-14}$ The purpose of our study was to determine whether ASL perfusion can detect alterations in CBF in patients with PHACE syndrome.

\section{MATERIALS AND METHODS Study Population}

This study was approved by the institutional review boards at each of the 3 hospitals (University of California, San Francisco; University of Pennsylvania; Medical College of Wisconsin), which waived the requirement for informed consent. Electronic medical records were reviewed from the 3 institutions for all patients with PHACE syndrome who underwent MR imaging with ASL from 2000 to 2019. All patients were discussed in the vascular anomalies clinic or in subspecialized pediatric dermatology clinics for cutaneous vascular anomalies at the respective institutions, where the diagnosis of PHACE syndrome was rendered using the latest PHACE syndrome consensus guidelines. ${ }^{9}$ Many patients were treated with propranolol for hemangioma, though this information was not fully available on each patient and these data were not collected in this study.

\section{ASL Technique}

Details of the ASL imaging technique varied depending on the institution, MR imaging vendor, and type of ASL acquisition scheme. ASL performed on 3T MR imaging systems (GE Healthcare) included pseudocontinous labeling with a background-suppressed 3D spiral acquisition with the following parameters: TR/TE = $4409 / 10.6 \mathrm{~ms}$; labeling period $=1500 \mathrm{~ms}$; postlabel delay $=$ $1500 \mathrm{~ms} ; \mathrm{FOV}=20 \mathrm{~cm}$; matrix $=128 \times 128$. ASL performed on 1.5 or $3 \mathrm{~T}$ (Siemens) MRI systems included $2 \mathrm{D}$ or $3 \mathrm{D}$ pulsed labeling with the following parameters: TR/TE $=2500 / 14 \mathrm{~ms}$; labeling period $=700 \mathrm{~ms}$; postlabel delay $=1800-1990 \mathrm{~ms} ; \mathrm{FOV}=20 \mathrm{~cm}$; matrix $=64 \times 64$. The postlabel delay on each MR imaging system was specified using the vendor's prefixed setting, and the parameter was not adjusted for age.

\section{Imaging Review}

Five neuroradiologists with $1,7,13,16$, and 16 years of postresidency experience in neuroimaging and arterial spin-labeling
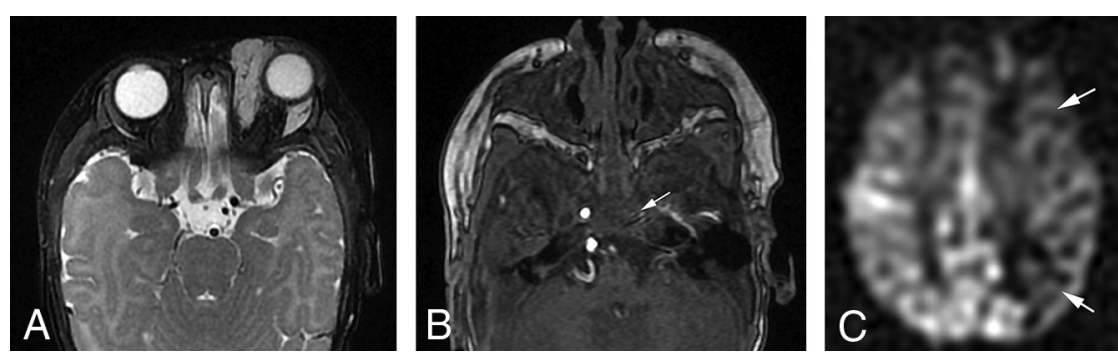

FIG 1. Decreased CBF in PHACE syndrome. A, Axial T2-weighted fat-suppressed image shows a left periorbital hemangioma. $B$, Axial time-of-flight MRA shows absence of the left cavernous ICA (arrow), which was reconstituted on the relatively more superior images (not shown). C, Axial ASL imaging shows decreased CBF signal in the left ICA territory (arrows). techniques and participation in a vascular anomalies clinic reviewed the MR images. CBF maps derived from ASL were qualitatively evaluated to determine whether there was decreased or normal perfusion within a major arterial territory. At least 1 neuroradiologist reviewed the images at each institution. In certain instances, 2 neuroradiologists assessed the imaging, and the evaluation was made by consensus. Arterial anomalies were characterized on the available noncontrast or contrast-enhanced MRA. The MRA images were evaluated in the same setting as the ASL images, and the neuroradiologists were not blinded to each set of images. The types of arteriopathies evaluated included the following: dysgenesis, narrowing, nonvisualization, persistent embryonic carotidvertebrobasilar arterial connections, and abnormalities in the arterial course and/or origin. ${ }^{3}$ The descriptor "dysgenesis" was used to describe bizarre looping, elongation, kinking, and aneurysmal enlargement. ${ }^{3}$ Parenchymal brain findings were assessed on DWI and T2/FLAIR, including the presence of acute or chronic infarct and any other developmental anomalies. The location of hemangiomas was also noted. If the hemangioma had involuted by the time of scanning, the location was recorded from the physical examination findings or previous assessments in the electronic chart.

\section{RESULTS}

\section{Patient Characteristics and ASL Findings}

Forty-one patients with PHACE syndrome had MR imaging examinations with ASL imaging (Online Table). There were 30 females (73\%) and 11 males (27\%). The ages ranged from 7 days to 15 years (median, 1 year; interquartile range, 3 months to 6.5 years). Of the 41 patients, 10 (24\%) had decreased CBF signal corresponding to a major arterial territory. Ten of 10 patients had decreased $\mathrm{CBF}$ signal in the anterior circulation, $2 / 10$ had decreased anterior and posterior circulation CBF signal, 2/10 had decreased bilateral anterior circulation CBF signal, and 1/10 had globally decreased CBF signal. Forty of 41 (97.5\%) patients had at least 1 arteriopathy, and in those with decreased CBF signal, the arteriopathy corresponded to the CBF signal alteration in $10 / 10$ patients. The 10 patients with decreased ASL signal had arteriopathies that demonstrated nonvisualization or narrowing of the major vessels supplying that vascular territory. Representative cases are seen in Figs 1-3.

\section{Conventional MR Imaging Findings}

None of the patients in this cohort had MR imaging evidence of acute or chronic infarct. Twenty-two of 41 (54\%) patients had posterior fossa abnormalities, such as cerebellar dysgenesis or Dandy-Walker malformation. Sixteen of 41 (39\%) patients had trigeminal cistern enlargement, which has been described in PHACE syndrome and is possibly related to aberrant migration of the cephalic neural crest in a metameric distribution. ${ }^{15}$ Fourteen of 41 (34\%) patients had hemangiomas identified on imaging, while the remaining hemangiomas had involuted by the time of scanning. The hemangiomas were ipsilateral to the arteriopathy in 16 of the 17 patients 

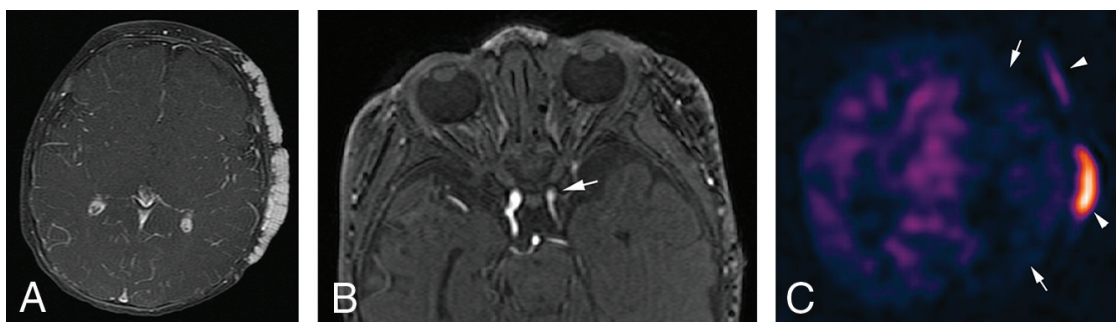

FIG 2. Decreased CBF in PHACE syndrome. A, Axial T1-weighted contrast-enhanced fat-suppressed image shows hemangiomas in the left frontoparietal scalp. $B$, Axial time-of-flight MRA shows a hypoplastic left cavernous ICA (arrow). C, Axial ASL imaging shows decreased CBF signal in the left ICA territory (arrows). The scalp hemangiomas show hyperintense signal (arrowheads) due to their arterial vascularity.
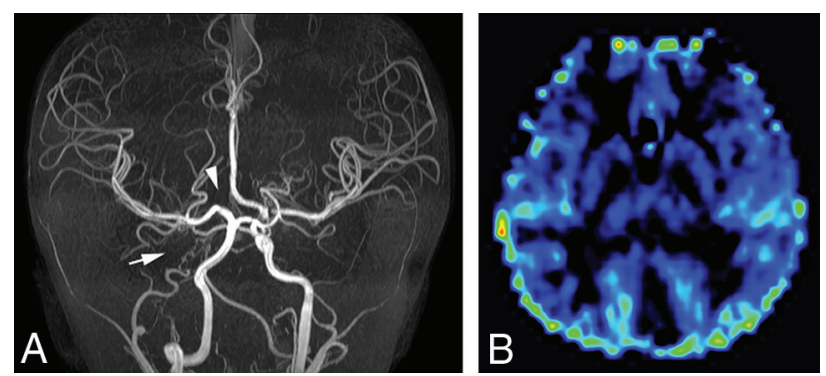

FIG 3. Normal CBF signal in PHACE syndrome. A, Time-of-flight MRA reconstruction shows absence of the right cervical and intracranial ICA (arrow). The right A1 anterior cerebral artery segment is also absent (arrowhead). B, Axial ASL imaging shows symmetric CBF bilaterally. This case shows that despite a severe arteriopathy, ASL provides additional value by showing normal CBF signal.

(94\%) with unilateral arteriopathy, which is in keeping with findings in another publication. ${ }^{3}$ The physical examination findings of these remaining hemangiomas are described in the Online Table.

\section{DISCUSSION}

To our knowledge, this multicenter study of 41 patients represents the largest cohort to date of patients with PHACE syndrome scanned with ASL perfusion imaging. While nearly every patient (40/41) had an arteriopathy, only approximately onequarter of patients (10/41) had decreased CBF signal in at least 1 major arterial territory. All patients with decreased CBF signal had involvement of the anterior circulation. None of the patients had acute or chronic infarction.

These findings suggest that ASL may serve a role in the imaging evaluation of patients with PHACE syndrome. The addition of ASL to the MR imaging/MRA protocol for PHACE can provide a potential physiologic assessment of stroke risk in future studies, as has been demonstrated in other intracranial arteriopathies. ${ }^{10,11}$ Relying solely on the structural arterial anomalies may overestimate the risk of stroke because the CBF may still be normal, given the collateral supply (Fig 3). This physiologic metric can potentially aid with risk stratification.

The risk of stroke in PHACE syndrome is not based solely on the complex arteriopathies but is likely multifactorial. Stroke risk may be potentially related to beta blocker use. Propranolol is the widely accepted first-line pharmacologic treatment agent for complicated infantile hemangiomas. ${ }^{16,17}$ Beta blockers could decrease cardiac output, reduce cerebral perfusion, and result in watershed infarcts, especially if there is a corresponding arteriopathy such as an absent vessel or severe stenosis. ${ }^{18}$ Increasing research related to propranolol therapy in PHACE syndrome has shown that the drug is well-tolerated with respect to stroke risk. A recent multicenter retrospective study of 76 patients treated with propranolol for infantile hemangioma in PHACE syndrome showed no serious adverse effect or stroke. ${ }^{18}$ Despite these reassuring data, stroke is still possible. One case report described an infant receiving propranolol at 11 months of age and developing a large right MCA infarct on MR imaging at 18 months of age. It is unclear whether the patient was still receiving propranolol at the time of the infarct, but the propranolol could have been a risk factor. ${ }^{5}$ ASL could be used as a helpful adjunct before propranolol therapy to help inform and potentially mitigate stroke risk.

In our practice, we have started to use the combination of ASL and MRA to provide a more complete picture of cerebrovascular hemodynamics in this group of patients before propranolol therapy. If there is a high risk of stroke based on CBF data and arterial anatomy, then propranolol is administered at the lowest possible dose and titrated judiciously in a monitored setting. Obviously, further research is needed to show the utility and efficacy of this practice. Hypoglycemia is another potential side effect of propranolol therapy for children with infantile hemangiomas. The mechanism is not fully understood but is possibly related to inhibition of $\beta$-adrenergic-mediated glycogenolysis, gluconeogenesis, and lipolysis, which impairs glucose homeostasis. ${ }^{19}$

The pathogenesis of arteriopathies in PHACE syndrome is poorly understood. In addition, the genetics of PHACE syndrome has not been well-elucidated but is hypothesized to be secondary to low-level postzygotic variants. ${ }^{20}$ Despite a candidate potential locus identified on $7 \mathrm{q} 33$ in 2 individuals, ${ }^{21}$ more recent research suggests that the affected gene in PHACE syndrome has not been discovered because the variant allele frequency of the mosaic mutations is extremely low. ${ }^{20}$ Further analysis with next-generation sequencing may provide causative mutations in PHACE syndrome.

ASL can provide additional assessment in PHACE beyond analysis of CBF. Hemangiomas are vascular tumors and show markedly hyperintense signal on ASL (Fig 2C). ${ }^{12-14}$ While hemangiomas are usually well seen on postcontrast T1 imaging, particularly with the use of fat suppression, ASL could increase lesion conspicuity for improved hemangioma detection, especially if fat suppression is not used or in locations that could be overlooked such as the internal auditory canals. ${ }^{22}$ Another advantage of ASL is its noncontrast technique. While we generally administer contrast to all patients with PHACE being scanned, ASL could also be performed to evaluate deep hemangiomas if contrast cannot be given or if refused by the patient or family. A sample MR imaging/MRA PHACE syndrome protocol is listed in the Table. 


\begin{tabular}{|c|c|}
\hline Sequence & Feature Evaluated \\
\hline Coronal and axial T2 fat-suppressed head and neck & $\begin{array}{l}\text { Facial hemangioma, extent of lesion, possible hemangioma in } \\
\text { airway }\end{array}$ \\
\hline Axial DWI brain & Acute ischemia \\
\hline Sagittal $\mathrm{Tl}$ or spoiled gradient-echo head and neck & Midline intracranial structures including pituitary gland, myelination \\
\hline Axial ASL head and neck & Cerebral blood flow, hemangioma \\
\hline Axial time-of-flight MRA head and neck & Arterial abnormalities \\
\hline Coronal contrast-enhanced dynamic MRA/MRV neck, if needed & Arterial abnormalities, including evaluation of the aortic arch \\
\hline Axial contrast-enhanced T1 fat-suppressed head and neck & Enhancement pattern \\
\hline $\begin{array}{l}\text { Coronal contrast-enhanced } \mathrm{Tl} \text { fat-suppressed internal auditory } \\
\text { canals }\end{array}$ & Hemangioma in the internal auditory canals \\
\hline
\end{tabular}

${ }^{a}$ Modified from Mamlouk et al ${ }^{13}$ with permission from Elsevier.

The utility and appearance of ASL imaging in cerebrovascular arteriopathies such as those seen in PHACE syndrome are dependent on a few factors that can potentially be a limitation if not properly recognized. Depending on the postlabel imaging time and severity of cerebrovascular stenosis, and hence arterial transit time, the appearance of ASL maps may be different. Typically, in singledelay ASL evaluations, the postlabel delay time is chosen so that by the time the brain is imaged, most of the label has ideally moved from the large arteries into the brain parenchyma, allowing more accurate parenchymal evaluation of CBF. In cases of arterial narrowing, slow flow, or flow through proximal collaterals, the label may still be mostly within the larger arteries and not yet transited into the parenchyma. There is also an additional confounder. The ASL label signal is T1-based and, therefore, has a very short time before inevitable longitudinal relaxation rapidly decreases the label brightness, which eventually loses signal very quickly and returns to normal. Therefore, in cases of more severe transit time delays, the low signal on ASL CBF maps may be due to the paucity of the remaining label in the assessed parenchyma rather than a true proportional decrease in $\mathrm{CBF}$. In these cases of severe transit time delays, the parenchymal CBF map may be more transit timeweighted than being truly CBF-weighted.

This underestimation of parenchymal CBF should be recognized in these physiologic states. This phenomenon can lead to errors and bias in the quantitative measurement of $\mathrm{CBF}$ in patients with PHACE syndrome with severe stenoses. Multidelay ASL, which is less commonly used and not commercially available from all MR imaging vendors, can partially mitigate this problem, though in severe arterial transit delay, this problem of underestimated CBF remains a potential problem. Velocity-selective ASL has been shown to be less sensitive to arterial transit delay inaccuracies of CBF measurement and may also be useful. ${ }^{23}$

Our study has limitations, including its retrospective nature. Different ASL techniques were used across our various institutions. Although these may be perceived as a limitation, use of different ASL techniques may potentially be a strength as well because it demonstrates the utility of the technique across a range of imaging schemes. On the other hand, differing parameters such as the labeling period and postlabel delay time could result in different CBF appearances in the same patient. In addition, these labeling periods and postlabel delays were not changed when scanning neonates versus children, the former having faster heart rates, possibly requiring a different postlabel delay. ${ }^{24}$ Another limitation is that because $\mathrm{CBF}$ changes were evaluated qualitatively, there was a subjective component in the assessment. However, the neuroradiologists in this study were experienced in the use and limitations of ASL for cerebrovascular disease evaluation. Finally, the lack of propranolol data in our study is another limitation of the assessment on whether the decreased CBF signal in some of our patients was due to PHACE syndrome or propranolol or both. Further research is needed in this specific patient cohort.

\section{CONCLUSIONS}

This study of 41 patients with PHACE syndrome shows the potential role of ASL imaging in demonstrating the hemodynamic changes in this patient group. In this cohort, only one-quarter of the studied patients had decreased CBF corresponding to a major arterial distribution, and none of the patients had acute or chronic ischemia. In all patients with decreased $\mathrm{CBF}$, the arteriopathies demonstrated nonvisualization or narrowing of the major vessels supplying that vascular territory. Further studies are needed to validate these results.

Disclosures: Christopher P. Hess—UNRELATED: Consultancy: Focused Ultrasound Foundation, Comments: personal fees, Data and Safety Monitoring Board member; Expert Testimony: various legal firms, Comments: personal fees, medical expert witness; Payment for Lectures Including Service on Speakers Bureaus: EUROKONGRESS GmBH, Comments: professional conference travel; Travel/Accommodations/ Meeting Expenses Unrelated to Activities Listed: Siemens, Comments: research travel; Other: GE Healthcare, Comments: personal fees, consultant.

\section{REFERENCES}

1. Siegel DH, Tefft KA, Kelly T, et al. Stroke in children with posterior fossa brain malformations, hemangiomas, arterial anomalies, coarctation of the aorta and cardiac defects, and eye abnormalities (PHACE) syndrome: a systematic review of the literature. Stroke 2012;43:1672-74 CrossRef Medline

2. Drolet BA, Dohil M, Golomb MR, et al. Early stroke and cerebral vasculopathy in children with facial hemangiomas and PHACE association. Pediatrics 2006;117:959-64 CrossRef Medline

3. Hess CP, Fullerton HJ, Metry DW, et al. Cervical and intracranial arterial anomalies in 70 patients with PHACE syndrome. AJNR Am J Neuroradiol 2010;31:1980-86 CrossRef Medline

4. Heyer GL, Millar WS, Ghatan S, et al. The neurologic aspects of PHACE: case report and review of the literature. Pediatr Neurol 2006;35:419-24 CrossRef Medline

5. Jack AS, Chow MM, Fiorillo L, et al. Bilateral pial synangiosis in a child with PHACE syndrome. J Neurosurg Pediatr 2016;17:70-75 CrossRef Medline

6. Jernigan S, Storey A, Hammer C, et al. Moyamoya syndrome and PHACE syndrome: clinical and radiographic characterization of the intracranial arteriopathy and response to surgical revascularization. J Neurosurg Pediatr 2019;23:493-97 CrossRef Medline 
7. Metry DW, Garzon MC, Drolet BA, et al. PHACE syndrome: current knowledge, future directions. Pediatr Dermatol 2009;26:381-98 CrossRef Medline

8. Haggstrom AN, Garzon MC, Baselga E, et al. Risk for PHACE syndrome in infants with large facial hemangiomas. Pediatrics 2010;126: e418-26 CrossRef Medline

9. Garzon MC, Epstein LG, Heyer GL, et al. PHACE syndrome: consensus-derived diagnosis and care recommendations. J Pediatr 2016;178:24-33.e22 CrossRef Medline

10. Haller S, Zaharchuk G, Thomas DL, et al. Arterial spin labeling perfusion of the brain: emerging clinical applications. Radiology 2016;281:337-56 CrossRef Medline

11. Lee S, Yun TJ, Yoo RE, et al. Monitoring cerebral perfusion changes after revascularization in patients with Moyamoya disease by using arterial spin-labeling MR imaging. Radiology 2018;288:565-72 CrossRef Medline

12. Mamlouk MD, Hess CP. Arterial spin-labeled perfusion for vascular anomalies in the pediatric head and neck. Clin Imaging 2016;40:104046 CrossRef Medline

13. Mamlouk MD, Nicholson AD, Cooke DL, et al. Tips and tricks to optimize MRI protocols for cutaneous vascular anomalies. Clin Imaging 2017;45:71-80 CrossRef Medline

14. Boulouis G, Dangouloff-Ros V, Boccara O, et al. Arterial spin-labeling to discriminate pediatric cervicofacial soft-tissue vascular anomalies. AJNR Am J Neuroradiol 2017;38:633-38 CrossRef Medline

15. Wright JN, Wycoco V. Asymmetric Meckel cave enlargement: a potential marker of PHACES syndrome. AJNR Am J Neuroradiol 2017;38:1223-27 CrossRef Medline

16. Krowchuk DP, Frieden IJ, Mancini AJ, et al; Subcommittee on The Management of Infantile Hemangiomas. Clinical practice guideline for the management of infantile hemangiomas. Pediatrics 2019;143: e20183475 CrossRef Medline

17. Léaute-Labrèze C, Boccara O, Degrugillier-Chopinet C, et al. Safety of oral propranolol for the treatment of infantile hemangioma: a systematic review. Pediatrics 2016;138:e20160353 CrossRef Medline

18. Olsen GM, Hansen LM, Stefanko NS, et al. Evaluating the safety of oral propranolol therapy in patients with PHACE syndrome. JAMA Dermatol 2020;156:186-90 CrossRef Medline

19. Holland KE, Frieden IJ, Frommelt PC, et al. Hypoglycemia in children taking propranolol for the treatment of infantile hemangioma. Arch Dermatol 2010;146:775-78 CrossRef Medline

20. Siegel DH. PHACE syndrome: infantile hemangiomas associated with multiple congenital anomalies-clues to the cause. Am J Med Genet C Semin Med Genet 2018;178:407-13 CrossRef Medline

21. Mitchell S, Siegel DH, Shieh JT, et al. Candidate locus analysis for PHACE syndrome. Am J Med Genet A 2012;158a:1363-67 CrossRef Medline

22. Mamlouk MD, Zimmerman B, Mathes EF, et al. Hearing loss in PHACE syndrome: clinical and radiologic findings. Childs Nerv Syst 2018;34:1717-24 CrossRef Medline

23. Bolar DS, Gagoski B, Orbach DB, et al. Comparison of CBF measured with combined velocity-selective arterial spin-labeling and pulsed arterial spin-labeling to blood flow patterns assessed by conventional angiography in pediatric Moyamoya. AJNR Am J Neuroradioly 2019;40:1842-49 CrossRef Medline

24. Alsop DC, Detre JA, Golay X, et al. Recommended implementation of arterial spin-labeled perfusion MRI for clinical applications: a consensus of the ISMRM perfusion study group and the European consortium for ASL in dementia. Magn Reson Med 2015;73:102-16 CrossRef Medline 